\title{
Oscillator-based assistance of cyclical movements: model-based and model-free approaches
}

\author{
Renaud Ronsse $\cdot$ Tommaso Lenzi $\cdot$ Nicola Vitiello $\cdot$ Bram Koopman • \\ Edwin van Asseldonk · Stefano Marco Maria De Rossi - Jesse van den Kieboom • \\ Herman van der Kooij · Maria Chiara Carrozza · Auke Jan Ijspeert
}

Received: 23 February 2011/Accepted: 30 July 2011/Published online: 1 September 2011

(C) International Federation for Medical and Biological Engineering 2011

\begin{abstract}
In this article, we propose a new method for providing assistance during cyclical movements. This method is trajectory-free, in the sense that it provides user assistance irrespective of the performed movement, and requires no other sensing than the assisting robot's own encoders. The approach is based on adaptive oscillators, i.e., mathematical tools that are capable of learning the high level features (frequency, envelope, etc.) of a periodic input signal. Here we present two experiments that we recently conducted to validate our approach: a simple sinusoidal movement of the elbow, that we designed as a proof-of-concept, and a walking experiment. In both cases, we collected evidence illustrating that our approach indeed assisted healthy subjects during movement execution. Owing to the intrinsic periodicity of daily life movements involving the lower-limbs, we postulate that our approach holds promise for the design of innovative rehabilitation
\end{abstract}

R. Ronsse · J. van den Kieboom · A. J. Ijspeert

Biorobotics Laboratory, Institute of Bioengineering, École Polytechnique Fédérale de Lausanne (EPFL), 1015 Lausanne, Switzerland

R. Ronsse $(\square)$

Centre for Research in Mechatronics (MCTR), Institute of Mechanics, Materials, and Civil Engineering, Université catholique de Louvain, 1348 Louvain-la-Neuve, Belgium e-mail: renaud.ronsse@uclouvain.be

T. Lenzi · N. Vitiello · S. M. M. De Rossi - M. C. Carrozza The BioRobotics Institute, Scuola Superiore Sant'Anna, 56025 Pontedera, Pi, Italy

B. Koopman - E. van Asseldonk · H. van der Kooij Biomechanical Engineering Laboratory, Institute for Biomedical Technology and Technical Medicine (MIRA), University of Twente, 7500 EA Enschede, The Netherlands and assistance protocols for the lower-limb, requiring little to no user-specific calibration.

Keywords Adaptive oscillator - Assistance - EMG · Exoskeleton $\cdot$ Metabolic cost $\cdot$ Walking

\section{Introduction}

In modern robotics research, a lot of attention is devoted to service applications, with the general objective to improve the human daily life [39]. In particular, assistive and rehabilitation robots have been proposed as an innovative mean to improve the condition of people affected by chronic or momentary movement disabilities [9, 13].

Assistive and rehabilitation robots have different goals. The former aims at assisting people affected by chronic movement disorders or neural lesions by providing continuous support giving extra power [20] or increasing movement accuracy [32]. On the other hand, the latter aims to retrain the nervous system and/or the musculoskeletal apparatus of the patient to restore his/her normal movement ability [27, 45].

Despite of having different objectives, human-robot interfacing is a critical issue for both assistive and rehabilitation robotics. The human-robot interface is indeed responsible for both power transfer and information transmission. More in detail, the human-robot physical interface is intended to provide a safe and comfortable interaction while transferring power between the two agents. Ergonomics studies [41] provide the main design guidelines for these interfaces, which are particularly critical in the case of wearable robots [7], due to the close interaction with the user. The human-robot cognitive interface instead is deputed to the acquisition and transfer of information regarding 
the cognitive involvement of the patient in the task (e.g., planning, reasoning, execution of a movement).

In the case of assistive robots, the goal is typically to amplify the movement initiated by the user, so that the effort spent by him/her is reduced without losing the control of the movement. On the contrary, rehabilitation robots exploit the information about the user intention to define the rehabilitative task in terms of spatio-temporal movement features. As such, the active participation of the patient in the task is promoted, and his/her effort is increased. This rehabilitative control strategy, commonly referred as "assist-as-needed", has been proved to be an effective way to increase the outcome of robot-mediated rehabilitation therapy by promoting motor recovery $[1,11,12,45,54]$.

In this article, we describe a new approach that we recently developed to estimate the user's intended movement while performing a cyclical motion task. This method can be used for both assistive and rehabilitative purposes. Unlike other methods previously used to estimate intended movements, our approach does not rely on inspecting activations by means of direct interfaces at the level of the central or peripheral nervous system or by electromyography (EMG) [7, 21, 22, 36]. EMG-based control has been successfully used to reduce the metabolic cost of walking of a healthy person [38], or to provide full-body daily assistance [20]. However, EMG recordings suffer from some drawbacks related to signal stability [6], which leads to the need of periodic recalibration and may also cause discomfort to the user over long periods of time (e.g., due to skin irritation). Our method requires no other sensing than the encoder of the robot actuators, avoiding the problems related to sensor placement, user-dependent calibration, or signal durability and reliability. As a consequence, our method provides both a fast and convenient integration to the user's body and an adaptivity to the user's intentions which-pending a sound and attractive ergonomic design —are the major requirements to maximize the device acceptability for potential users.

To compensate for the "loss" of information that could have been provided by direct sensing of the user status (e.g., EMGs), we embedded some a-priori knowledge about the movement directly into the controller. In the case of lowerlimb movements, this a-priori knowledge simply consisted of assuming the movement to be periodic, a hallmark of daily life activities involving the lower-limbs (walking, running, stair climbing, etc.). The strategy proposed here exploits the concept of motor primitives, which emerged from biology $[2,16]$ and has now clearly percolated in robotics $[8,17]$. The concept of motor primitives is very general in neuroscience, since motor primitives were identified at the cerebral, spinal, muscular, and kinematic levels. Nonetheless, the underlying idea of motor primitives is that a complex motor behavior can be described as the composition of simpler building blocks (i.e., the motor primitives) by using a finite set of parameters. The proposed movement estimation method follows this principle: Instead of directly estimating the intended movement kinematics (the epiphenomenon of the intended movement), we make use of the a-priori knowledge that the movement is periodic to derive a non-linear dynamical system able to represent the movement in a finite set of simple features. Specifically, we make use of adaptive oscillators $[4,29]$, a mathematical tool capable of synchronizing to a periodic signal and extracting its relevant features (like its frequency and envelope) through dynamical equations.

In this article, we present the results of two recent experiments. Experiment 1 was conceived as a proof-ofconcept of the whole approach. For that reason, we designed this experiment to be as simple as possible: we focused on sinusoidal movements about the elbow joint. As such, we avoided the intrinsic complexities related to the lower-limb, like complex periodic joint profiles, multijoints coordination, and contacts with the ground. Nonetheless, we asked the participants to perform the movement around the vertical position, mimicking the inverted pendulum configuration of the leg during the stance phase of walking [15]. This experiment was already published in $[31,33,34]$ and is only surveyed here. Experiment 2 extends the approach to walking assistance, and therefore specifically addresses the related challenges. Preliminary results were recently published in [35]. Both experiments deal with movement assistance of healthy participants. Therefore, we recorded biological signals-namely EMGs and oxygen consumption-illustrating that less effort (or energy) was required from the participants to perform the same movement, in steady-state regime. We further paid particular attention to design conditions illustrating the adaptive features of our controller, i.e., requiring the participants to modulate their limb trajectory. This last point is explored in conditions involving transient behavior, i.e., changes in the movement pattern. Extension of our approach to rehabilitation protocols involving patients will be an intensive field for future research.

\section{Methods}

\subsection{Feature extraction of a sinusoidal input using an adaptive oscillator}

The central element of the movement assistance approaches presented in this paper is an adaptive oscillator, a tool developed by Righetti et al. [4, 29] and used in many applications [30, 34]. Here, we introduce the simplest adaptive oscillator, which can be regarded as an augmented phase oscillator: 
$\dot{\phi}(t)=\omega(t)+v F(t) \cos \phi(t)$,

where, $\phi(t)$ is the oscillator phase, $\omega(t)$ its intrinsic frequency, and $v$ the learning parameter determining the speed of phase synchronization to the periodic teaching signal $F(t)$. In order to learn the frequency of the teaching signal $F(t)$, instead of doing mere synchronization only, the oscillator frequency is turned into a new state variable, integrating the phase update:

$\dot{\omega}(t)=v F(t) \cos \phi(t)$.

As such, Righetti et al. developed an adaptive oscillator, having the capacity to constantly adapt its intrinsic frequency to the teaching signal frequency, and to keep this input frequency in memory, i.e., in the state variable $\omega(t)$.

Let us now assume (i) that the input signal follows a sinusoidal pattern, i.e., $\theta(t)=\alpha_{1 \text {,in }} \sin \left(\omega_{\text {in }} t\right)+\alpha_{0, \text { in }}$, where $\alpha_{1, \text { in }}, \omega_{\text {in }}$, and $\alpha_{0, \text { in }}$ are the amplitude, frequency, and offset of this input, respectively; and (ii) that the adaptive oscillator (1), (2) is synchronized with this input. As a consequence, an observer of $\theta(t)$ can be obtained by:

$\hat{\theta}(t)=\alpha_{1}(t) \sin \phi(t)+\alpha_{0}(t)$

where $\alpha_{1}(t), \phi(t)$, and $\alpha_{0}(t)$ are supposed to converge to the corresponding input variables. Righetti et al. [29] showed that this convergence is guaranteed by using the difference between the input $\theta(t)$ and the filtered (or estimated) input $\hat{\theta}(t)$ as teaching signal: $F(t)=\theta(t)-\hat{\theta}(t)$, and by implementing the following integrators for learning the amplitude and offset:

$\dot{\alpha}_{0}(t)=\eta F(t), \quad \dot{\alpha}_{1}(t)=\eta F(t) \sin \phi(t)$,

where $\eta$ is the integrator gain. Again, (2) and (4) reach steady-state when $F(t)=0$, i.e., when $\hat{\theta}(t)=\theta(t)$. If $\theta(t)$ is only quasi-sinusoidal-i.e., if $\alpha_{1, \text { in }}, \omega_{\text {in }}$, and $\alpha_{0 \text {,in }}$ slowly vary in time- $\hat{\theta}(t)$ will be a low-pass filtered version of $\theta(t)$, but importantly, both will still be phase-synchronized on average [6]. This is a critical difference between this approach and classical low-pass filtering, which unavoidably introduces delay.

In sum, the adaptive oscillator presented above provides a continuous estimate of the input signal features: frequency (2), amplitude, and offset (4). It further provides a filtered version of the input (3) which has, on average, the same phase as the input (see the example in Fig. 1).

\subsection{Survey of experiment 1: assistance of a simple elbow cyclical movement}

In [34], we used the adaptive oscillator presented above to assist a simple cyclical movement of the elbow around the vertical (upright) position. This was a model-based approach, in the sense that an inverse dynamic model of the assisted joint was used to retrieve and amplify the torque provided by the participant. For all details about this experiment, the reader is referred to $[33,34]$.

\subsubsection{Movement assistance}

We use the elbow position as input $\theta(t)$ of the adaptive oscillator, and assume it to be (quasi-)sinusoidal. As such, Eqs. 2 and 4 provide not only a zero-delay smooth estimate of the input signal (3), but also of its velocity and acceleration:

$\hat{\dot{\theta}}(t)=\alpha_{1}(t) \omega(t) \cos \phi(t)$,

$\hat{\ddot{\theta}}(t)=-\alpha_{1}(t) \omega(t)^{2} \sin \phi(t)$.

Let's now assume that the elbow dynamics can be captured with a simple dynamical model $\Sigma$ :

$\ddot{\theta}(t)=\Sigma(\theta(t), \dot{\theta}(t), u(t), \Pi)$,

where $\Pi$ represents the forearm parameters. An estimate of the total torque $\hat{u}(t)$ applied to the elbow joint can be retrieved based on an inverse dynamic model $\Sigma_{i n v}$, i.e.:

$\hat{u}(t)=\Sigma_{\text {inv }}(\hat{\theta}(t), \hat{\dot{\theta}}(t), \hat{\ddot{\theta}}(t), \Pi)$.

Finally, $\hat{u}(t)$ is the estimate of the input torque $u(t)$ that is applied at the elbow joint, both by the user $u_{h}(t)$ and by the assistance device $u_{e}(t)$, i.e., $u(t)=u_{h}(t)+u_{e}(t)$.

Assistance is provided by feeding back a fraction of this estimated torque to the user, i.e.:

$u_{e}(t)=\kappa \hat{u}(t)$,

with the level of assistance $0 \leq \kappa<1$. Assuming a stationary sinusoidal movement and a perfect inverse dynamical model (6), such that $\hat{u}(t)=u(t)$, the total torque

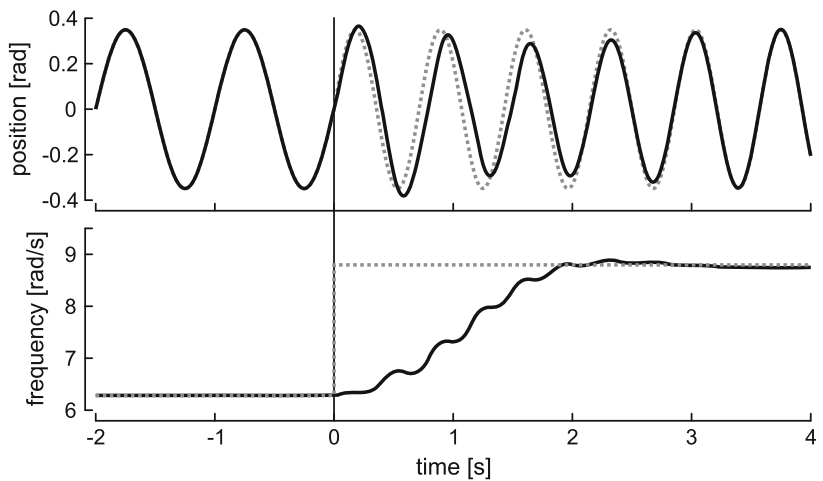

Fig. 1 Example of the oscillator's adaptation dynamics. Top The oscillator's output $\hat{\theta}(t)$ (solid black line) filters out the sudden change in the input $\theta(t)$ (dotted gray line), i.e., a frequency step at $t=0$. Bottom Corresponding evolution of the learned frequency $\omega(t)$. Adapted with permission from Ronsse et al. 34 (ㄷ 2011 IEEE) 
should emerge from a collaboration between the user (performing $100(1-\kappa) \%$ of the effort) and the assistance device (performing $100 \kappa \%$ of the effort).

Parameters used in Experiment 1 were equal to $v=20$ (1), (2), and $\eta=5$ (4).

\subsubsection{Experimental protocol}

The assistance device we used in this experiment was the NEUROExos, an active elbow orthosis conceived for neurorehabilitation and assistance purposes [23]. Participants were asked to put their forearm in the upright vertical position, and to make a cyclical flexion/extension movement around this position. Movement pace was driven by a metronome (one full flexion-extension cycle between two consecutive beeps).

Each participant underwent three types of condition, in the following order:

1. "no-exo": the NEUROExos was actually replaced by a simple 1-dof goniometer. This was a control condition.

2. "constant frequency": in this condition, the target movement pace was constant and equal to $1 \mathrm{~Hz}$. Participants performed consecutive trials having a different level of assistance ( $\kappa$ in (7)): $\kappa=0, \kappa=$ $0.33, \kappa=0$ (wash-out), $\kappa=0.5$, and $\kappa=0$ (wash-out) (see Fig. 5a for the succession of trials).

3. "variable frequency": to illustrate that participants had the possibility to modulate the movement features, we introduced a condition where the target movement pace varied across the trials (see [34] for details). Participants again performed consecutive trials with the same succession of assistance levels as in the "constant frequency" condition.

In order to monitor the participant's effort, associated with movement performance during all conditions, we recorded the surface EMG activity from the biceps brachii and triceps brachii muscle.

\subsection{Experiment 2: walking assistance}

In the second experiment, we extended the approach described above to walking assistance. However, two important new challenges appeared in this transfer: (i) the joint trajectory can no longer be considered as being sinusoidal, such that (3) would not provide reliable estimate of the trajectory; (ii) the inverse model of the legs during walking would be much more complicated to derive as it was for the elbow, for instance due to the intermittent interactions with the ground. Therefore, in contrast to Experiment 1, we propose here a model-free approach for walking assistance.

\subsubsection{Real-time filtering of non-sinusoidal but periodic signals}

If the input signal is periodic but non-sinusoidal, Righetti et al. [28] proposed to extend the method explained in Sect. 2.1 by putting several oscillators in parallel (see the upper part of Fig. 2). As such, each of these oscillators should learn one frequency component of the input signal, providing therefore a kind of real-time Fourier decomposition. We slightly adapted the equations of [28] by assuming that the input signal was periodic. Therefore, only the fundamental frequency had to be learned, the others being multiples of it. Concretely, (1), (2), and (4) were changed to:

$$
\begin{aligned}
\dot{\phi}_{i}(t) & =i \omega(t)+v F(t) \cos \phi_{i}(t), \\
\dot{\omega}(t) & =v F(t) \cos \phi_{1}(t), \\
\dot{\alpha}_{i}(t) & =\eta F(t) \sin \phi_{i}(t),
\end{aligned}
$$

with $F(t)=\theta(t)-\hat{\theta}(t), \hat{\theta}(t)=\sum_{i=0}^{K} \alpha_{i}(t) \sin \phi_{i}(t)$, and $i \in$ $[0, K]$ are the $K+1$ parallel oscillators. Note that, in (8), the 0th oscillator is still a simple integrator, learning the input offset, with $\phi_{0}(t)=\phi_{0}(0)=\pi / 2$.

However, with the decomposition presented above, a large number of oscillators was still required to properly learn dwell intervals within the joint signals, like, e.g., the plateau in the knee profile during the stance phase of walking. To improve the quality of the signal estimate, we coupled the pool of adaptive oscillators to a kernel-based non-linear filter, similarly to [14] (Fig. 2). Note however

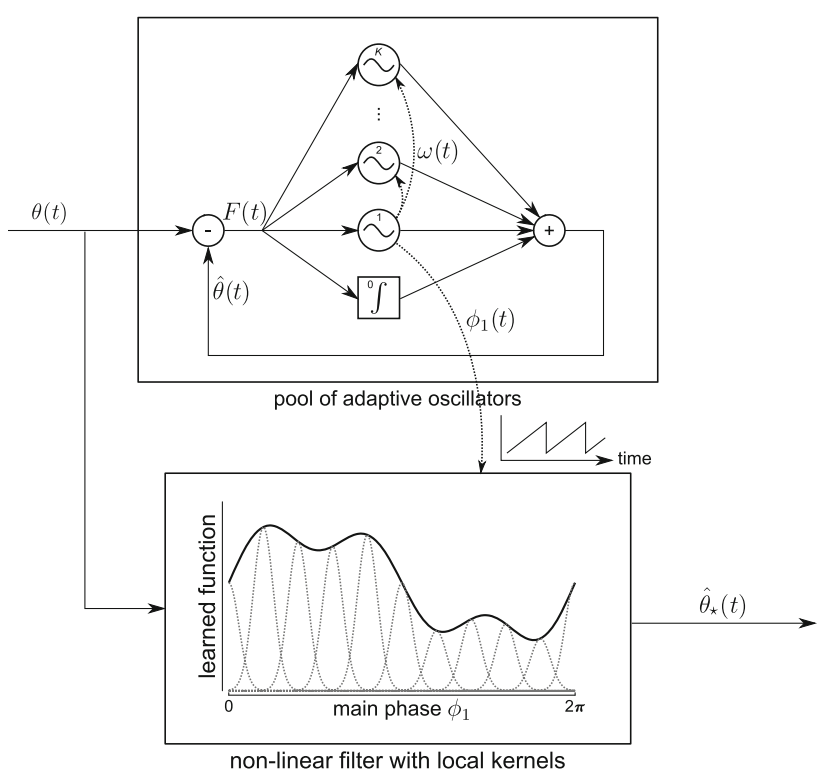

Fig. 2 Online learning of a periodic but non-sinusoidal input signal $\theta(t)$. The upper block is a pool of adaptive oscillators (1), (4), decomposing the input into a real-time Fourier series. The lower block is a kernel-based non-linear filter, mapping the phase of the main harmonic $\phi_{1}(t)$ to the input envelope. Adapted from [14] 
that we simplified the derivations made in [14] since our application did not target imitation learning, but only filtering.

This approach can be formulated as a supervised learning problem:

$\hat{\theta}_{\star}(t)=\frac{\sum \Psi_{i}\left(\phi_{1}(t)\right) w_{i}}{\sum \Psi_{i}\left(\phi_{1}(t)\right)}$,

where $\Psi_{i}(\phi(t))=\exp \left(h\left(\cos \left(\phi(t)-c_{i}\right)-1\right)\right)$ is a set of $N$ Gaussian-like kernel functions. The parameter $h$ determines their width, and $c_{i}$ their center (equally spaced between 0 and $2 \pi$ in $N$ steps). This algorithm then constructs a series of local mappings of the input $\theta(t)$ as a function of the phase $\phi_{1}(t)$, and an estimate of the input $\hat{\theta}_{\star}(t)$ from a weighted sum of these mappings.

Following [14, 40], an online version of this learning process can be implemented using incremental regression, which is done with the use of recursive least squares with a forgetting factor of $\lambda$, to determine the weights $w_{i}$. Given the target data $\theta(t), w_{i}$ is updated by:

$$
\begin{aligned}
& w_{i}(k+1)=w_{i}(k)+\Psi_{i}(k) P_{i}(k+1)\left(\theta(k)-w_{i}(k)\right), \\
& P_{i}(k+1)=\frac{1}{\lambda}\left(P_{i}(k)-\frac{P_{i}(k)^{2}}{\frac{\lambda}{\Psi_{i}(k)}+P_{i}(k)}\right),
\end{aligned}
$$

where $P$ is the inverse covariance matrix [24]. If $\lambda<1$, the regression gives more weight to recent data. Figure 3 shows the performance of this filter during a representative cycle in steady-state regime. In sum, three parameters determine the speed of convergence of the algorithm: $v$ and $\eta$ are related to the adaptive oscillator and therefore establish the convergence rate to frequency changes, and $\lambda$ tunes the time constant for modulations in the pattern envelope.

\subsubsection{Model-free assistance}

In order to provide assistance without relying on an inverse dynamical model of the body, we adopted the following approach. First, the system presented in Sect. 2.3.1 was used to provide an estimate of the joint(s) position in the future. Indeed, using (9) by replacing $\phi(t)$ by $\phi(t)+\Delta_{\phi}$, an estimate of what the joint position should be at a time corresponding to a $\Delta_{\phi}$ phase lead in the future can be derived:

$$
\begin{aligned}
\hat{\theta}_{\star, \Delta}(t) & =\frac{\sum \Psi_{i, \Delta}\left(\phi_{1}(t)\right) w_{i}}{\sum \Psi_{i, \Delta}\left(\phi_{1}(t)\right)}, \\
\Psi_{i, \Delta}(\phi(t)) & =\exp \left(h\left(\cos \left(\phi(t)+\Delta_{\phi}-c_{i}\right)-1\right)\right) .
\end{aligned}
$$

Again, Fig. 3 shows an example, where $\hat{\theta}_{\star, \Delta}$ is a good prediction of the future joint trajectory. Second, this

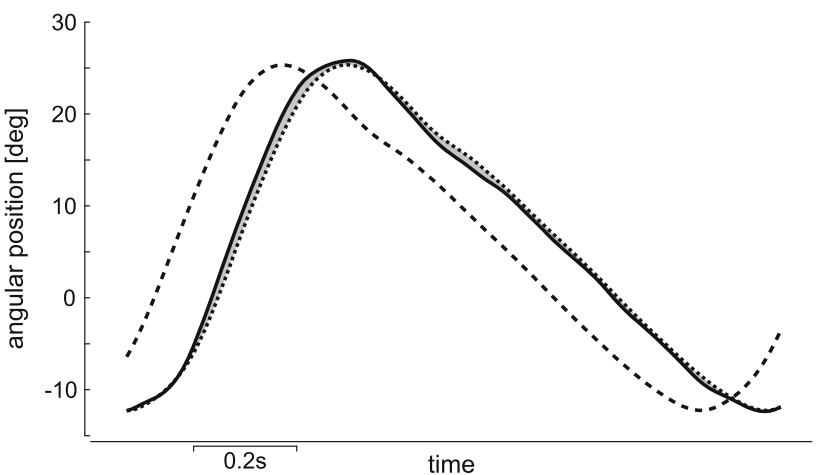

Fig. 3 Right hip trajectory during a representative cycle of the "high assistance" condition. The figure shows the actual joint trajectory $\theta$ (solid), the filtered trajectory $\hat{\theta}_{\star}$ from the kernel filter (9) (dotted), and the trajectory $\hat{\theta}_{\star, \Delta}$ predicted by the kernel filter (11) (dashed)

estimated future position $\hat{\theta}_{\star, \Delta}(t)$ can be used to attract the user's joint in a force field:

$u_{e}(t)=k_{f}\left(\hat{\theta}_{\star, \Delta}(t)-\theta(t)\right)$,

where $k_{f}$ is the field stiffness and $u_{e}(t)$ the desired torque to be applied by the assistive device.

In sum, the method of assistance we implemented here is aiming at continuously attracting the user's joints to their own future (using the force field (11), (12)), but leaving the opportunity to the user to constantly adapt the frequency (through the adaptive oscillator (8)) and shape (through the filter (10)) of this attractive pattern. For simplicity, we used a constant stiffness $k_{f}$ and no damping in the force field (12), and we assisted only the two hips using two separate force fields. Both sides were coupled together by forcing the oscillators amplitudes and frequency (8) to reach a consensus (averaging).

Parameters used in Experiment 2 were equal to: $v=$ $6, \eta=0.25, K=6, \lambda=0.9999, N=90, h=144, \quad$ and $\Delta_{\phi}=36^{\circ}(10 \%$ of the cycle $)$.

\subsubsection{Participants and experimental setup}

Nine healthy participants took part in Experiment 2 (aged 24-28, weight 58-86, four females, five males). The experiments were conducted in agreement with the local institution's ethics regulations, and participants signed a written consent form.

For testing our approach, we used the LOPES (see Fig. 4a), a treadmill-based lower-limb exoskeleton developed at the University of Twente [44, 45, 47, 48], and capable of assisting 8 DOFs of the lower-limbs (right and left hip abduction/adduction, hip flexion/extension, and knee flexion/extension, forward/backward and sideways movements of the pelvis) by providing torques through the 

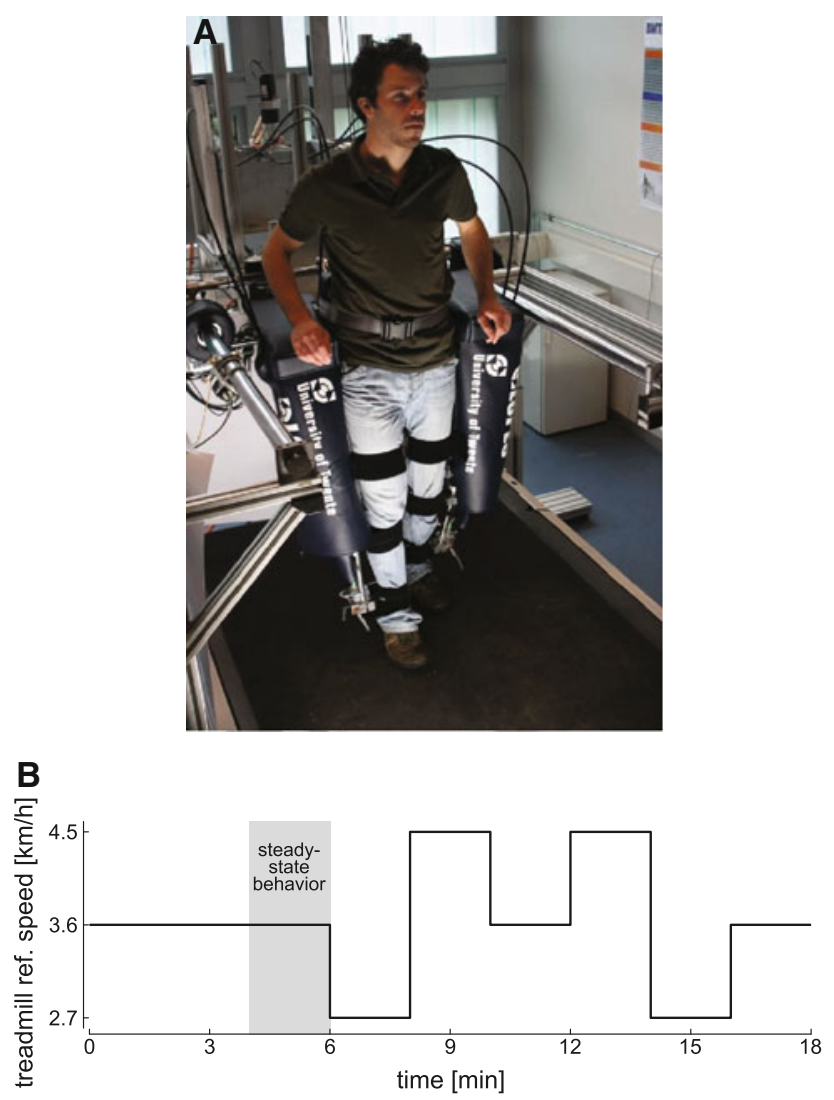

Fig. 4 a Picture of an healthy subject wearing the LOPES. b Variations of the treadmill reference speed over a typical trial of "low assistance" or "high assistance" condition. During the first $6 \mathrm{~min}$, the reference speed was invariably equal to $3.6 \mathrm{~km} / \mathrm{h}$. The last 2 min of this initial plateau (shaded area) correspond to steady-state behavior for the analyses. During the last $12 \mathrm{~min}$, the reference speed was made of 2-min long plateaus at slow $(2.7 \mathrm{~km} / \mathrm{h})$, normal $(3.6 \mathrm{~km} / \mathrm{h})$, and fast $(4.5 \mathrm{~km} / \mathrm{h})$ speed. Transitions were randomized for each trial

principle of series elastic actuation [26, 44]. The LOPES is lightweight and actuation is produced remotely by means of Bowden cables. Therefore, it is considered as a close-totransparent device, inducing only small changes in the kinematic and EMG patterns with respect to normal walking [48].

The joint kinematics were recorded using the LOPES sensors, both to feed the adaptive oscillators, and to proceed with post-hoc analyses. The LOPES was controlled using Matlab (the Mathworks, Natick, MA), with a sampling time of $1 \mathrm{~ms}$.

The energy expended by the participants in the various conditions was measured by the Oxycon Pro system (Jaeger, Hoechberg, Germany). Participants were connected to the Oxycon with a flexible tube making an airtight seal to a facemask, measuring oxygen consumption $\left(V_{\mathrm{O}_{2}}\right)$ and the volume expiration $\left(V_{E}\right)$. Every five seconds $(0.2 \mathrm{~Hz})$ these parameters were measured and stored on the personal computer connected to the Oxycon. Thereafter, the normalized rate of expended energy was inferred from the formula used in [3]:

$\bar{E}[\mathrm{~W} / \mathrm{kg}]=\frac{16.58 \dot{V}_{\mathrm{O}_{2}}+4.51 \dot{V}_{\mathrm{CO}_{2}}}{W}$,

where $\dot{V}_{\mathrm{O}_{2}}$ and $\dot{V}_{\mathrm{CO}_{2}}$ are the rates of $\mathrm{O}_{2}$ and $\mathrm{CO}_{2}$ volume involved in respiratory exchange, and $W$ is the participant body weight.

\subsubsection{Experimental protocol}

The participant walked comfortably on the treadmill, wearing the LOPES on both legs, except during the "free walking" condition, detailed later. The LOPES was fastened via attachment cuffs to the middle of the thighs, and the top and bottom of the calves (see Fig. 4a). The LOPES pelvis module was further attached to the participant waist with a belt.

This study focused on assistance in the sagittal plane, and we decided to assist only the hips during walking. To improve the LOPES transparency, a force proportional to the joints' speed was applied to the hips and knees to compensate for the friction induced by the exoskeleton's joints.

Each participant underwent four types of condition, in a randomized order:

1. "free walking": participants walked on the treadmill without wearing the LOPES. This condition lasted a single trial of about $6 \mathrm{~min}$, and was used to evaluate the rate of expended energy during normal walking.

2. "transparent": the LOPES was controlled to be as transparent as possible, i.e., by setting $k_{f}=0$ in (12), for both hips. This condition lasted a single trial of about $6 \mathrm{~min}$, and was used to evaluate the actual level of transparency of the LOPES on gait cadence and energy consumption.

3. "low assistance": participants received an assistance of $k_{f}=0.0142 W \mathrm{Nm} / \mathrm{deg}$ at the hips, where $W$ is the participant's total body weight. This condition consisted of two trials: the first one lasted about $6 \mathrm{~min}$ at a constant treadmill speed $(3.6 \mathrm{~km} / \mathrm{h})$, and the second one lasted about 12 min with treadmill speed variations (see Fig. 4b).

4. "high assistance": participants received an assistance of $k_{f}=0.0284 \mathrm{~W} \mathrm{Nm} / \mathrm{deg}$ at the hips. This condition also consisted of two trials (constant and variable treadmill speed) being 6 and 12 min long.

Note that the two levels of assistance were calculated based on pilot results, to provide, on average, an absolute assisitive torque corresponding to 50 and $100 \%$ of the average absolute torque produced by the hip during walking, as reported in [53]. 


\subsection{Data analysis and statistics}

For both experiments, most of the variables analyzed in the Results section were computed for each movement cycle, delimited by movement reversals in Experiment 1 and left heel strikes in Experiment 2, using an appropriate separation algorithm based on kinematics landmarks and force plate data (for Experiment 2). Statistical significance of the changes induced by different modes of assistance was evaluated using $n$-ways and repeated measures ANOVAs. For all significant effects, post-hoc tests (Bonferroni adjustment) were performed. All data processing and statistics were computed using Matlab, and with a $P$ factor of 0.05 .

\section{Results}

\subsection{Steady-state kinematic profiles}

This section presents the main variations that were observed in the kinematic patterns in both experiments, depending whether assistance was provided or not. It focuses on steady-state behavior, i.e., the last 20 cycles for each condition in Experiment 1, and the shaded area of Fig. $4 \mathrm{~b}$ in Experiment 2.

In Experiment 1, the kinematic profiles stayed very similar across the different conditions. For example, the average duration of movement cycles is shown in Fig. 5a for the "no-exo" condition and the "constant frequency" condition. The figure reveals a transient effect due to the adaptation to the assistance torque: the cycle duration rapidly decreased (corresponding to faster movements) and reached again the target pace after about 5-10 cycles. Some transients are also visible at the beginning of the "wash-out" trials (3 and 5), but they disappeared more rapidly. We performed statistics on the steady-state performance with the condition/level of assistance as the unique factor, which revealed no variation in the steadystate cycle duration. Similar results were obtained for the movement amplitude [36].

Regarding Experiment 2, cycle duration did show a modulation depending on the level of assistance, even in steady-state, i.e., at the end of the 6-min long plateaus with $3.6 \mathrm{~km} / \mathrm{h}$ of treadmill reference speed: the higher the assistance, the faster the cycles (see Table 1). Repeated measures ANOVA reached significance, with post-hoc tests establishing a significant difference between the "high assistance" condition and the two unassisted conditions. On top of cycle duration modulations, changes also appeared in the kinematic profiles, as shown in Fig. 6 for the right leg (they are very similar, but with a phase-shift of $50 \%$, for the left one). The figure shows that, when
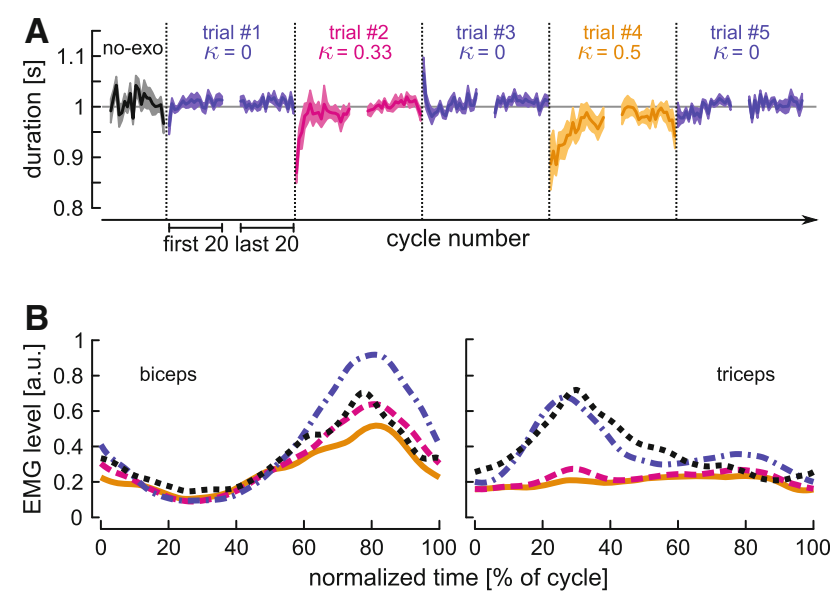

Fig. 5 Main results from the "constant frequency" condition in Experiment 1 (adapted with permission from [34], (C) 2011 IEEE). a Cycle-by-cycle evolution of the cycle duration. The figure shows the last 20 cycles of the "no-exo" condition, and the first and last 20 cycles of each trial of the "constant frequency" condition. Shaded areas represent the between-participants SEM. b Steady-state EMG profiles (biceps, left; triceps, right), averaged across participants. These profiles were obtained by resampling the actual trajectories over 101 equally spaced points for each cycle, then averaging for each of the 101 points

assistance was provided, the movement tended to be more ample, even for the knee (which was never assisted). Table 1 gives the movement range values, and repeated measures ANOVA always reached significance. In sum, the higher the assistance, the ampler the movements. In contrast, the joint angles remained more or less invariant at the time of contact with the ground (i.e., around $50 \%$ of the cycle). This means that, with assistance, the joints clearly overshot during swing the position to be reached at impact, creating an unecessary offset in the trajectory (shown for the hip in Fig. 6).

\subsection{Evidences of assistance}

This section presents the results establishing that our assistive methods indeed facilitated the movement execution, again focusing on steady-state behavior.

In Experiment 1, this was done by recording the biceps and triceps EMG during task execution. Figure 5b shows the steady-state EMG profiles normalized over the whole cycle. Two important results are visible on this figure: (i) wearing the exoskeleton without assistance (difference between the "no-exo" condition and the "constant frequency" condition with $\kappa=0$ ) induced larger biceps activity, this being certainly due to the exoskeleton forearm's mass and inertia, which were not compensated in that mode and which mainly loaded the joint flexor; and (ii) providing assistance (both $\kappa=0.33$ and $\kappa=0.5$ ) progressively induced a marked decrease in peak EMG. The 
Table 1 Relevant variables during steady-state behavior of Experiment 2. Given values are mean \pm SEM

\begin{tabular}{llllll}
\hline Variable & \multicolumn{2}{l}{ Values } & & \multirow{2}{*}{ Rep. meas. ANOVA } \\
\cline { 2 - 5 } & "Free walking" & "Transparent" & "Low assitance” & "High assitance” & \\
\hline Cycle duration (s) & $1.24 \pm 0.02$ & $1.24 \pm 0.02$ & $1.20 \pm 0.01$ & $1.14 \pm 0.02$ & $P(3,24)=6.77, P=0.002$ \\
Right hip movement range $\left({ }^{\circ}\right)$ & - & $38.8 \pm 1.3$ & $41.1 \pm 1.4$ & $46.7 \pm 1.6$ & $F(2,16)=21.8, P=0.0001$ \\
Left hip movement range $\left({ }^{\circ}\right)$ & - & $40.4 \pm 1.4$ & $42.5 \pm 1.1$ & $47.5 \pm 1.2$ & $F(2,16)=19.3, P=0.0001$ \\
Right knee movement range $\left({ }^{\circ}\right)$ & - & $52.9 \pm 1.6$ & $59.8 \pm 1.8$ & $69.3 \pm 2.1$ & $F(2,16)=142.8, P<0.0001$ \\
Left knee movement range $\left({ }^{\circ}\right)$ & - & $58.8 \pm 1.7$ & $65.2 \pm 1.2$ & $72.7 \pm 1.1$ & $F(2,16)=54.7, P<0.0001$ \\
Norm. transferred power $(\mathrm{W} / \mathrm{kg})$ & - & $-0.45 \pm 0.02$ & $9.58 \pm 0.37$ & $26.38 \pm 1.24$ & $F(2,16)=194.4, P<0.0001$ \\
\hline
\end{tabular}

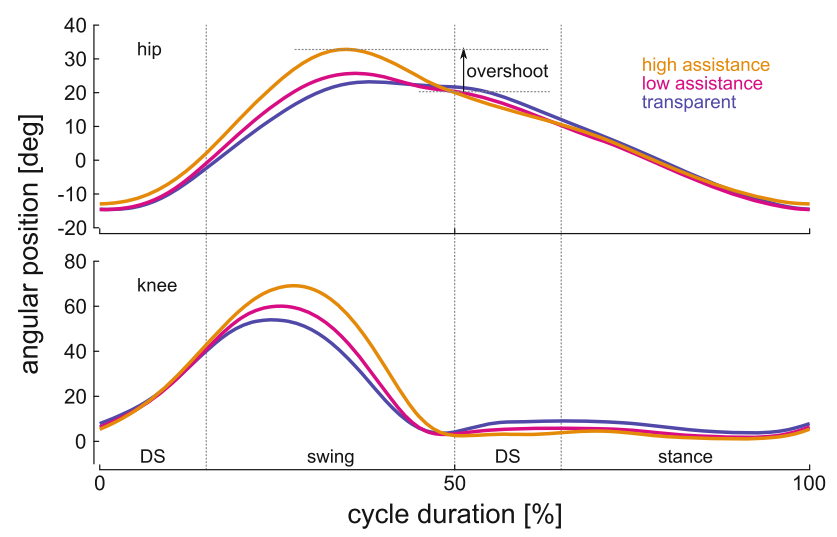

Fig. 6 Angular trajectory of the right hip (top) and knee (bottom) during the "transparent" condition (blue), the "low assistance" condition (purple), and the "high assistance" condition (orange), in steady-state, with a walking speed of $3.6 \mathrm{~km} / \mathrm{h}$. Labels show the periods of double support (DS), swing, and single support stance. Averaged across participants

highest level of assistance we tested $(\kappa=0.5)$ corresponded to a decrease of about $26 \%$ in the biceps peak EMG, and 59\% in the triceps peak EMG with respect to the "no-exo" condition.

Regarding Experiment 2, evidence that assistance was provided to the participants will be established in two steps. First, we measured the average power (i.e., torque $\times$ velocity), normalized by the body weight, transmitted by the LOPES to the participant. While this quantity obviously oscillated around $0 \mathrm{~W} / \mathrm{kg}$ in the "transparent condition", it raised up to higher values in both assisted conditions (see Table 1). Interestingly, we found a correlation between this amount of power transfer at the hip and the offset in hip trajectory illustrated in Fig. 6, among the different participants. Figure 7 shows this relationship during steady-state performance of the "high assistance" condition, and establishes that those who "tolerated" the largest offset in their hip trajectory were those who received the largest amount of power from the device.

Second, the level of assistance was also directly assessed by computing the metabolic energy consumption, from (13). Figure 8 shows this result. First, it is visible that the supposedly transparent exoskeleton actually significantly loaded the participants, since the rate of expended energy increased from the "free walking" to the "transparent" condition. Second, the figure establishes the efficiency of the assistance, since the rate of expended energy decreased back for the two assisted conditions, to about two thirds of the difference with the "free walking" condition. Interestingly, by computing the rate of expended energy during the very last minute of the "low assistance" and "high assistance" conditions (which always corresponded to a "normal" treadmill reference speed, see Fig. 4b), we observed a more important decrease, as if the long period with variable treadmill speed helped the participants to get more and more familiar with the provided assistance. The repeated measure ANOVA confirmed the significance of this modulation $(F(5,35)=7.5, p=0.0001)$.

\subsection{Adaptivity}

One of the main properties and advantages of the oscillator-based approach presented in this article is its capacity to adapt to movement changes induced by the user. Data validating this capacity to adapt are presented in this section. Therefore, this section specifically refers to transitory phases, i.e., the "variable frequency" condition in Experiment 1 and the last 12 minutes of the "low assistance" and "high assistance" conditions in Experiment 2.

In Experiment 1, adaptivity was explored in a dedicated condition, namely the "variable frequency" condition, where the participants were asked to modulate their movement frequency throughout each trial. Participants succeeded in achieving this frequency modulation, and the adaptive oscillator managed to track the constantly changing input frequency. Interestingly, we found again a decrease of the biceps and triceps EMG when assistance was provided, showing a facilitation of the movement even when the movement pace was not stationary $[33,34]$.

Owing to the very large time constant of metabolic adaptation, it was not possible to measure the assistance efficiency during the variable speed phase of the "low assistance" and "high assistance" conditions of Experiment 


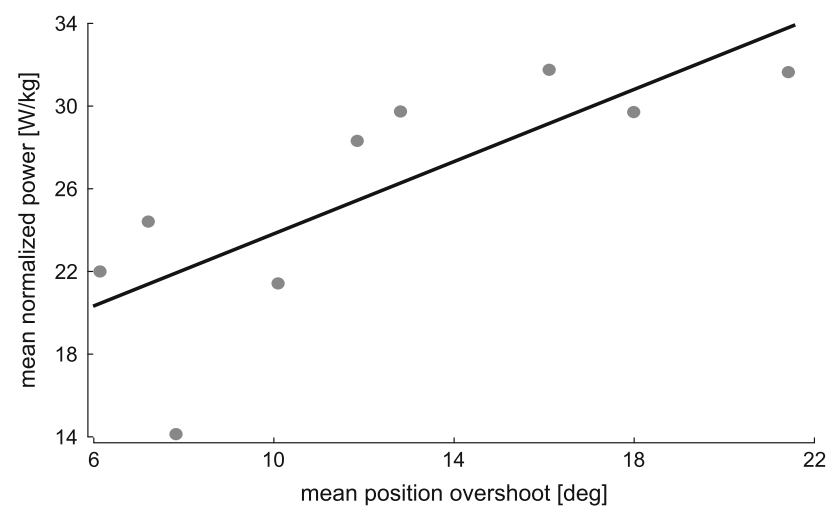

Fig. 7 Correlation between the amount of trajectory offset and the average power transfered from the device to the participant, for the right hip, and in steady-state during the "high assistance" condition. Each point represents an individual participant, and the solid line represents the correlation slope. Correlation coefficient: $r=0.77$ $(p<0.015)$

2. Therefore, for this experiment, we will rather focus on a characterization of the time constants of adaptation of the assistive algorithm. Figure 9 shows the time evolution of different variables around a representative variation in the treadmill reference speed (here, from slow to fast speed). After the transition, the movement pace changed rapidly, and this change was almost instantaneously detected by the adaptive oscillator. The error in estimated position (gray area in Fig. 3) took a bit more time to converge back to the steadystate value. Thereafter, the power transferred from the device to the user also converged to steady-state. To quantify these trends, we fitted exponential curves on these data, for all participants, all transitions, and both levels of assistance. More precisely, we fitted an equation of the form $\gamma_{1}(1-$ $\left.e^{-c / \delta_{\mathrm{s}}}\right)+\gamma_{0}$ on the actual and estimated frequencies, and on the transferred power, and of the form $\gamma_{1}\left(1-e^{-c / \delta_{\mathrm{f}}}\right) e^{-c / \delta_{\mathrm{s}}}+$

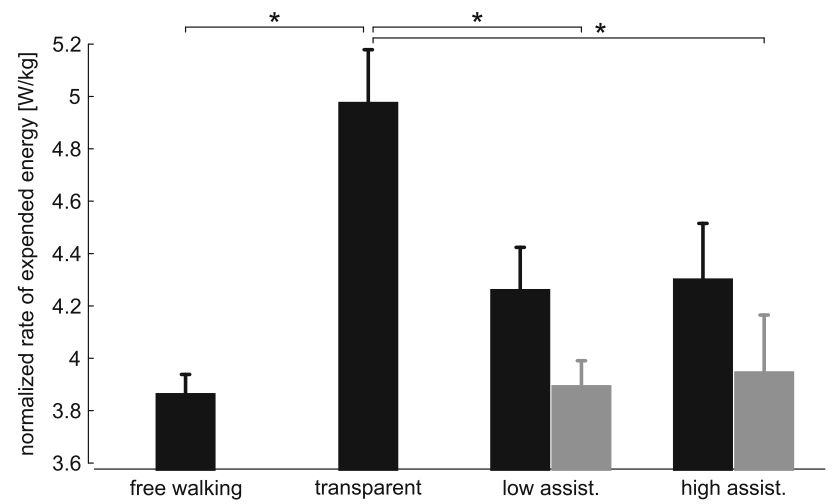

Fig. 8 Normalized rate of expended energy by the participants during the different conditions, in steady-state. The gray bars represent the data recorded at the very end (i.e., during the last minute) of the "low assistance" and "high assistance" conditions. Error bars represent the between-participants SEM. Stars show the pairwise comparisons reaching significance (post-hoc) $\gamma_{0}$ on the position error, where $c$ corresponds to the cycle number, with $c=0$ at the transition. For the analysis, we kept the $\delta_{\mathrm{s}}$ 's as the (slowest) time constants of interest. Said differently, if the identified time constant was $\delta_{\mathrm{s}}$, the corresponding variable took $\delta_{\mathrm{s}}$ cycles to reach $67 \%$ of its new steady-state value.

Figure 9e shows these time constants, for the 6 possible transitions and the "low assistance" and "high assistance" conditions. Obviously, the fastest one corresponded to the adaptation of the actual movement frequency, and varied between less than one cycle and around six cycles, depending on the transition and condition. Note that this time constant cannot be compared with the time constant of the actual treadmill speed adaptation, since actual treadmill speed was not recorded. Very consistently, the estimated frequency adapted about one cycle later, this delay being solely caused by the oscillator dynamics (8). The position error adapted with a slower time constant of about six cycles, corresponding to the adaptation of the kernel filter (10). The normalized transferred power evolved in parallel since, as suggested by (12) the transferred torque directly depended on the estimated position $\hat{\theta}_{\star}(t)$. First, a repeated measures ANOVA with the variable as single factor confirmed the causality: $\delta_{\text {act_freq }}<\delta_{\text {est_freq }}<\delta_{\text {pos_err }} \simeq \delta_{\text {pow }}(F(3,321)=$ $46.6, P<0.0001)$. Second, looking to each variable independently, we designed Two-way repeated measures ANOVAs (6 transitions $\times 2$ conditions) to sort out these dependences:

The time constant of adaptation of the actual movement frequencies depended on the transition type $(F(5,40)=$ $3, P=0.02)$, on the condition $(F(1,40)=23.8, P=$ $0.001)$, and on their interaction $(F(5,40)=3.2, P=0.02)$.

The time constant of adaptation of the estimated movement frequencies depended also on the transition type $(F(5,40)=2.6, P=0.04)$, and on the condition $(F(1,40)=18.8, P=0.003)$, but not on their interaction $(P>0.2)$.

The time constant of adaptation of the position error depended also on the type of transition $(F(5,10)=$ $2.5, P=0.05)$, and on the condition $(F(1,40)=6.5, P=$ $0.03)$, but not on their interaction $(p>0.5)$.

The time constant of adaptation of the transferred power depended only on the transition type $(F(5,40)=9.9$, $P<0.0001$ ), but neither on the condition, nor on their interaction (both $P$ 's $>0.3$ ).

Figure 9e shows that, in general, all time constants were smaller (i.e., faster adaptation) in transitions from a faster to a slower treadmill speed. Moreover, adaptation of the movement frequency tended to go faster for the "low assistance" than for the "high assistance" condition.

In sum, this analysis shows a causality in the adaptation of our assistive algorithm: the estimated frequency adapted 


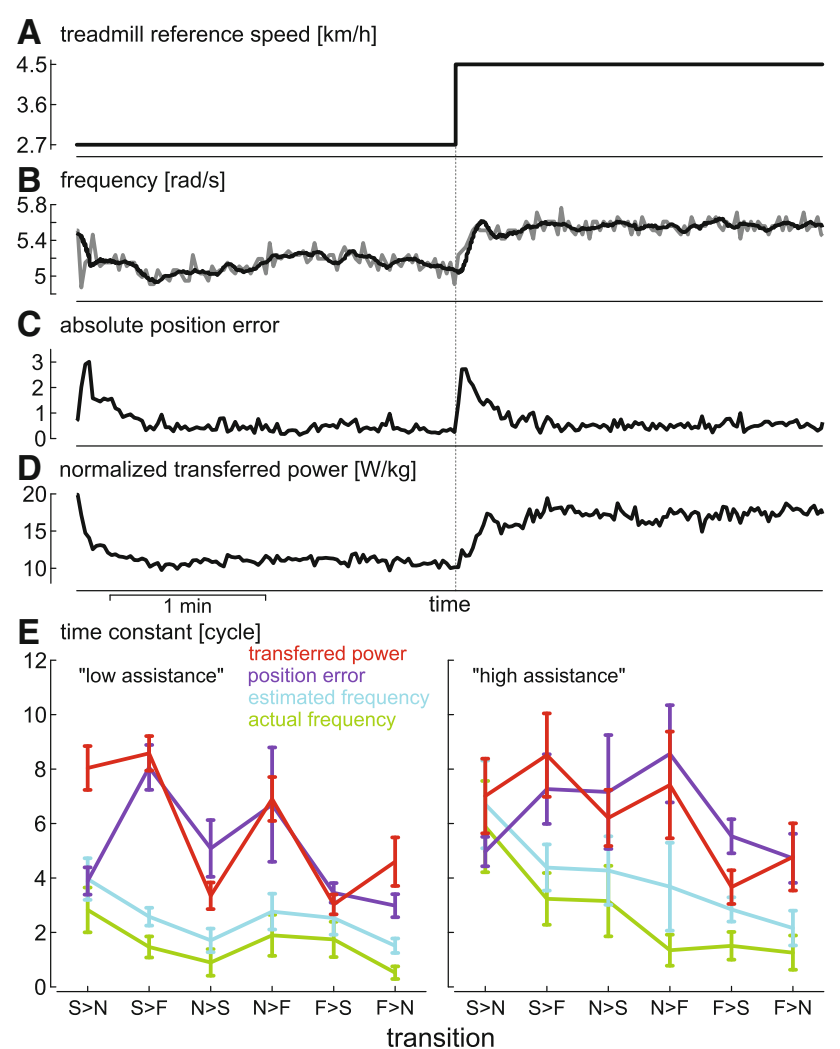

Fig. 9 For a representative participant and transition, the figure shows the time evolution of different variables around a variation in the treadmill reference speed (a), b actual movement frequency (inverse of the cycle duration, gray) and movement frequency estimated by (8) (black); $\mathbf{c}$ absolute error in estimated position, i.e., $\left\|\theta(t)-\hat{\theta}_{\star}(t)\right\|$, where $\hat{\theta}_{\star}(t)$ is given by (9) (gray area in Fig. 3); and $\mathbf{d}$ normalized power transferred from the device to the user. $\mathbf{c}$ and d are averaged over each cycle. e Slowest time constant of the return to steady-state of the actual and estimated movement frequency, the position error, and the transferred power. Values are given for the right hip (when applicable), the six possible transitions, and the "low assistance" and "high assistance" conditions. Error bars represent the between-participants SEM

rapidly after the actual change in movement pace, and the kernel adaptation followed later, corresponding to a new steady-state in the transferred power. All time constants were below ten cycles-i.e., about $12 \mathrm{~s}$-an encouraging result to envision real-life scenarios.

\section{Discussion}

This article presented two experiments that we recently conducted to validate a new approach to assisting cyclical movements using adaptive oscillators. We described three main results: (i) some subtle changes in the movement pattern due to the assistance, (ii) evidence that the assistance indeed reduced the muscular activity or the metabolic cost during the task execution, and (iii) the adaptive features of our mechanism.
The main characteristic of our approach is its capacity to achieve a robust synchronization between the user and the assistive robot. Synchronization is an ubiquitous phenomenon in biology [42] and, during locomotion, is observed at the level of spinal central pattern generators to guarantee the pattern coordination [18]. Here, we proposed to implement the synchronization mechanisms by means of adaptive oscillators [4, 29, 30] such that, on top of inputoutput synchronization, the movement features (amplitude, frequency, etc.) are stored in dedicated state variables. This turned out to be very helpful to derive both model-based and -free algorithms. In Experiment 1, we developed a model-based strategy based on a real-time estimate of the trajectory profile (including first and second order derivatives), and in Experiment 2, we used the estimated state variables to make a prediction about the future joints position, in a model-free version.

Kinematic analysis focused on potential changes in the movement pattern, depending on whether assistance was provided or not. In Experiment 1, only minor changes were found in steady-state behavior. This is logical, since the movement pace was driven by a metronome, and the pattern was quite stereotyped. In Experiment 2, more interesting changes were observed. First, we observed an increase of the overall task tempo (cycle frequency) when assistance was provided. Consequently, the stride length decreased, to maintain a constant forward speed (imposed by the treadmill). The type and level of assistance that the participants received were driven by dynamical systems and consequently, the perceived leg impedance can likely be altered by the assistance. For example, in the framework of Experiment 1 , it is easy to show that an "ideal" controller should decrease the perceived mass, inertia, and friction coefficient of the assisted limb by a factor $\kappa$. This is more difficult to quantify for Experiment 2, due to the absence of a dynamical model, but similar effects should be in place. Therefore, the resonance frequency of the coupled (leg and actuated robot) system could also vary with the level of assistance, and the participant could intend to change the gait cadence to match with the changing resonance frequency [50, 52]. As documented in the literature (e.g., [53]), the pattern shape slightly changes with the cadence, a phenomenon that we observed here as well.

The main variation in the gait pattern was the appearance of an offset in the hip trajectory before heel strike. Said differently, during the swing phase, the whole leg moved "too much" forward, requiring a small backward movement before heel strike. Interestingly, the participants who made the largest offsets were also those who received the largest amount of power (normalized to their body weight) from the device. This offset could thus be viewed as an adaptation of the walking gait to maximize the received assistance. 
Both in Experiments 1 and 2, we paid attention to collect data to validate that our approach indeed facilitated the movement execution, by lowering the human effort associated to it. These data corresponded to biometric variables (EMGs in Experiment 1, and oxygen consumption in Experiment 2), and were thus completely decoupled from the robot controllers. The corresponding analyses tended to validate our assumption, although, in Experiment 2, we only managed to reach a level slightly above the one during free walking. As such, the benefit of our assistance was completely washed out by the burden of wearing the device. These results are however encouraging, and they have to be balanced with the literature illustrating the challenge related to reduce the metabolic cost of free walking with an assistive device [9, 37, 38, 51]. Possible directions to improve this result would require to (i) make the LOPES more transparent [25, 46], (ii) increase the level of assistance, (iii) give longer familiarization trials to the users (Fig. 8 already suggests a further improvement at the very end of the trial), and (iv) develop a more sensible assistance scheme. Regarding this last point, it is worth noting that the force field implemented in Experiment 2 (12) basically provided a torque which is proportional to the joint velocity (since $\hat{\theta}_{\star, \Delta}(t)-\theta(t)$ approximates the curve tangent, and is then close to its first derivative). As a consequence, the provided power (torque $\times$ angular velocity) can only be positive (proportional to the squared velocity). This is maybe appropriate for the hip (which mostly delivers positive power during walking), but clearly not for the knee $[43,53]$. This could explain why pilot experiments showed that this type of assistance was not appropriate for the knee, suggesting us to only assist the hips in Experiment 2.

Finally, some results were also collected to illustrate the capacity of our controllers to adapt to changes in the steady-state movement pattern. These changes can be caused either by external factors (a change in the level of assistance-Experiment 1 -or in the treadmill speedExperiment 2), or internal factors (a voluntary modulation of the movement frequency-Experiment 1). In both experiments, we showed that the intrinsic adaptation time constant of the behavior was always smaller than ten cycles, and was actually equal to the time constant of the oscillator itself. As demonstrated in the "variable frequency" condition of Experiment 1, this permitted smooth adaptation to changes in movement frequency, as long as the rate of change stayed below the intrinsic time constant of the adaptive oscillator. Future investigations will be conducted to establish whether these time constants are compatible with real-life scenarios and, if necessary, how the oscillator's time constants should be tuned to deal with real-life requirements. Note that additional security mechanisms could be implemented to decrease the level of assistance during the non-stationary phase, for instance when the state variables first derivatives (8) are above a certain threshold.

This requirement to adapt the provided assistance depending on the user behavior is of prime interest in the framework of assist-as-needed rehabilitation robotics. We believe that the oscillator-based framework presented in this article nicely complements other approaches based on compliance [10, 27, 45, 47], adaptation [1, 19, 27] or adaptive learning of a dynamical model for the task at hand [54]. In particular, our approach relies on the concept of motor primitives, which first emerged in biology [2, 16] and is now extensively used in robotics [8, 17]. Assistance and rehabilitation robotics lie at the intersection of both fields, and should therefore be an ideal testbed for the concept. In particular, the concept of motor primitives postulates that some "knowledge" about the movement is hard-coded within the bottom layer of the controller, in order to decrease the bandwidth requirement between the "brain" and this bottom layer [18]. This is exactly what we did here, by pre-coding the fact that the task is cyclical (in both experiments) and sinusoidal (only in Experiment 1) into the controller. As such, no adaptation of the controller (i.e., no information transfer) was required during steadystate cyclical behavior.

The biggest challenge for us in the coming years will be to transfer these concepts to actual rehabilitation, i.e., to studies with patients. We believe that our approach opens up promising avenues, since (i) it provides assistance which is intuitive for the user (again, our naive healthy participants adapted as fast as the oscillator itself), (ii) it requires no sensor placement (only the robot joints position has to be sensed), (iii) it provides trajectory-free assistance, and (iv) it opens perspective to derive assist-as-needed protocols, for example by modulating the assistance gain as a function of the gait phase. Moreover, our framework could be easily adapted to different scenarios, or different patient needs, due to the intrinsic flexibility of dynamical systems.

In conclusion, this article presented a new method to provide movement assistance during rhythmic movements. Summarizing the results from $[33,34]$, the first experiment was a proof-of-concept, which was further extended to a walking task (Experiment 2). The main result we obtained is that our method indeed assisted the user during the movement execution, without preventing him/her to keep the full control on the movement features. Importantly, this was achieved using simple and cheap sensors, i.e., only the device encoders.

Acknowledgements The authors were funded by the EU within the EVRYON Collaborative Project STREP (FP7-ICT-2007-3-231451). 


\section{References}

1. Banala SK, Kim SH, Agrawal SK, Scholz JP (2009) Robot assisted gait training with active leg exoskeleton (alex). IEEE Trans Neural Syst Rehabil Eng 17(1):2-8

2. Bizzi E, Cheung VCK, d'Avella A, Saltiel P, Tresch M (2008) Combining modules for movement. Brain Res Rev 57(1): $125-133$

3. Brockway JM (1987) Derivation of formulae used to calculate energy expenditure in man. Hum Nutr Clin Nutr 41(6):463-471

4. Buchli J, Righetti L, Ijspeert AJ (2008) Frequency analysis with coupled nonlinear oscillators. Physica D 237:1705-1718

5. Cavallaro E, Rosen J, Perry J, Burns S (2006) Real-time myoprocessors for a neural controlled powered exoskeleton arm. IEEE Trans Biomed Eng 53(11):2387-2396

6. Dankaerts W, O'Sullivan PB, Burnett AF, Straker LM, Danneels LA (2004) Reliability of emg measurements for trunk muscles during maximal and sub-maximal voluntary isometric contractions in healthy controls and clbp patients. J Electromyogr Kinesiol 14(3):333-342

7. De Rossi SMM, Vitiello N, Lenzi T, Ronsse R, Koopman B, Persichetti A, Vecchi F, Ijspeert AJ, van der Kooij H, Carrozza MC (2011) Sensing pressure distribution on a lower-limb exoskeleton physical human-machine interface. Sensors 11(1):207-227

8. Dégallier S, Ijspeert A (2010) Modeling discrete and rhythmic movements through motor primitives: a review. Biol Cybern 103(4):319-338

9. Dollar A, Herr H (2008) Lower extremity exoskeletons and active orthoses: Challenges and state-of-the-art. IEEE Trans Robotics 24(1):144-158

10. Duschau-Wicke A, von Zitzewitz J, Caprez A, Lunenburger L, Riener R (2010) Path control: A method for patient-cooperative robot-aided gait rehabilitation. IEEE Trans Neural Syst Rehabil Eng 18(1):38-48

11. Edgerton VR, Roy RR (2009) Robotic training and spinal cord plasticity. Brain Res Bull 78(1):4-12

12. Emken J, Harkema S, Beres-Jones J, Ferreira C, Reinkensmeyer D (2008) Feasibility of manual teach-and-replay and continuous impedance shaping for robotic locomotor training following spinal cord injury. IEEE Trans Biomed Eng 55(1):322-334

13. Ferris DP (2009) The exoskeletons are here. J Neuroeng Rehabil 6:17-0

14. Gams A, Ijspeert AJ, Schaal S, Lenarčič J (2009) On-line learning and modulation of periodic movements with nonlinear dynamical systems. Auton Robot 27:3-23

15. Goswami A, Thuilot B, Espiau B (1998) A study of the passive gait of a compass-like biped robot: symmetry and chaos. Int $\mathbf{J}$ Robotics Res 17(12):1282-1301

16. Hart CB, Giszter SF (2010) A neural basis for motor primitives in the spinal cord. J Neurosci 30(4):1322-1336

17. Ijspeert A, Nakanishi J, Schaal S (2003) Learning attractor landscapes for learning motor primitives. In: Advances in neural Information processing systems, vol 15, MIT Press, Cambridge, pp 1547-1554

18. Ijspeert AJ (2008) Central pattern generators for locomotion control in animals and robots: a review. Neural Netw 21(4):642-653

19. Jezernik S, Colombo G, Morari M (2004) Automatic gait-pattern adaptation algorithms for rehabilitation with a 4-dof robotic orthosis. IEEE Trans Robot Autom 20(3):574-582

20. Kawamoto H, Sankai Y (2005) Power assist method based on phase sequence and muscle force condition for HAL. Advanced Robotics 19(7):717-734

21. Kiguchi K, Iwami K, Yasuda M, Watanabe K, Fukuda T (2003) An exoskeletal robot for human shoulder joint motion assist. Mechatronics, IEEE/ASME Transactions on 8(1):125-135
22. Kinnaird C, Ferris D (2009) Medial gastrocnemius myoelectric control of a robotic ankle exoskeleton. IEEE Trans Neural Syst Rehabil Eng 17(1):31-37

23. Lenzi T, De Rossi S, Vitiello N, Chiri A, Roccella S, Giovacchini F, Vecchi F, Carrozza MC (2009) The neuro-robotics paradigm: NEURARM, NEUROE xos, HANDEXOS. In: Proc. Annual International Conference of the IEEE Engineering in Medicine and Biology Society EMBC 2009, pp 2430-2433

24. Ljung L, Söderström T (1983) Theory and Practice of Recursive Identification. The MIT Press Signal Processing, Optimization, and Control Series, The MIT Press, Cambridge, Massachusetts

25. Nef T, Lum P (2009) Improving backdrivability in geared rehabilitation robots. Med Biol Eng Comput 47(4):441-447

26. Pratt GA, Williamson MM (1995) Series elastic actuators. In: Proc. IEEE/RSJ International Conference on Intelligent Robots and Systems 95. 'Human Robot Interaction and Cooperative Robots', vol 1, pp 399-406

27. Riener R, Lünenburger L, Jezernik S, Anderschitz M, Colombo G, Dietz V (2005) Patient-cooperative strategies for robot-aided treadmill training: first experimental results. IEEE Trans Neural Syst Rehabil Eng 13(3):380-394

28. Righetti L, Ijspeert AJ (2006) Programmable central pattern generators: an application to biped locomotion control. In: Proc. IEEE International Conference on Robotics and Automation ICRA 2006, pp 1585-1590

29. Righetti L, Buchli J, Ijspeert AJ (2006) Dynamic hebbian learning in adaptive frequency oscillators. Physica D 216:269-281

30. Righetti L, Buchli J, Ijspeert AJ (2009) Adaptive frequency oscillators and applications. The Open Cybernetics and Systemics Journal 3:64-69

31. Rinderknecht MD, Delaloye FA, Crespi A, Ronsse R, Ijspeert AJ (2011) Assistance using adaptive oscillators: Robustness to errors in the identification of the limb parameters. In: Rehabilitation Robotics, 2011 IEEE International Conference on, pp 210-215

32. Rocon E, Belda-Lois J, Ruiz A, Manto M, Moreno J, Pons J (2007) Design and validation of a rehabilitation robotic exoskeleton for tremor assessment and suppression. IEEE Trans Neural Syst Rehabil Eng 15(3):367-378

33. Ronsse R, Vitiello N, Lenzi T, van den Kieboom J, Carrozza MC, Ijspeert AJ (2010) Adaptive oscillators with human-in-the-loop: Proof of concept for assistance and rehabilitation. In: Biomedical Robotics and Biomechatronics (BioRob), 2010 3rd IEEE RAS and EMBS International Conference on, pp 668-674

34. Ronsse R, Vitiello N, Lenzi T, van den Kieboom J, Carrozza MC, Ijspeert AJ (2011) Human-robot synchrony: Flexible assistance using adaptive oscillators. IEEE Trans Biomed Eng 58(4):1001-1012

35. Ronsse R, Koopman B, Vitiello N, Lenzi T, De Rossi SMM, van den Kieboom J, van Asseldonk E, Carrozza MC, van des Kooij H, Ijspeert AJ (2011) Oscillator-based Walking Assistance: a Model-free Approach. In: Rehabilitation Robotics, 2011 IEEE International Conference on, pp 216-221

36. Rosen J, Brand M, Fuchs MB, Arcan M (2001) A myosignalbased powered exoskeleton system. IEEE Trans Syst, Man, Cybern A, Syst, Humans 31(3):210-222

37. Sawicki GS, Ferris DP (2008) Mechanics and energetics of level walking with powered ankle exoskeletons. J Exp Biol 211(Pt 9):1402-1413

38. Sawicki GS, Ferris DP (2009) Powered ankle exoskeletons reveal the metabolic cost of plantar flexor mechanical work during walking with longer steps at constant step frequency. J Exp Biol 212(Pt 1):21-31

39. Schaal S (2007) The new robotics-towards human-centered machines. HFSP Journal 1(2):115-26

40. Schaal S, Atkeson CG (1998) Constructive incremental learning from only local information. Neural Comput 10(8):2047-2084 
41. Schiele A, van der Helm F (2006) Kinematic design to improve ergonomics in human machine interaction. IEEE Trans Neural Syst Rehabil Eng 14(4):456-469

42. Strogatz SH (2003) Sync: The Emerging Science of Spontaneous Order. Hyperion, New York

43. Unal R, Behrens S, Carloni R, Hekman E, Stramigioli S, Koopman $H$ (2010) Prototype design and realization of an innovative energy efficient transfemoral prosthesis. In: Biomedical Robotics and Biomechatronics (BioRob), 2010 3rd IEEE RAS and EMBS International Conference on, pp $191-196$

44. Vallery H, Veneman J, van Asseldonk E, Ekkelenkamp R, Buss M, van Der Kooij H (2008) Compliant actuation of rehabilitation robots. IEEE Robotics Automation Magazine 15(3):60-69

45. Vallery H, van Asseldonk EHF, Buss M, van der Kooij H (2009) Reference trajectory generation for rehabilitation robots: complementary limb motion estimation. IEEE Trans Neural Syst Rehabil Eng 17(1):23-30

46. Vallery H, Duschau-Wicke A, Riener R (2009) Optimized passive dynamics improve transparency of haptic devices. In: Robotics and Automation, 2009. ICRA '09. IEEE International Conference on, pp 301-306

47. van Asseldonk EHF, Ekkelenkamp R, Veneman JF, Van der Helm FCT, van der Kooij H (2007) Selective control of a subtask of walking in a robotic gait trainer(lopes). In: Proc. IEEE 10th International Conference on Rehabilitation Robotics ICORR 2007, pp 841-848
48. van Asseldonk EHF, Veneman JF, Ekkelenkamp R, Buurke JH, van der Helm FCT, van der Kooij H (2008) The effects on kinematics and muscle activity of walking in a robotic gait trainer during zero-force control. IEEE Trans Neural Syst Rehabil Eng 16(4):360-370

49. Veneman JF, Kruidhof R, Hekman EEG, Ekkelenkamp R, Asseldonk EHFV, van der Kooij H (2007) Design and evaluation of the lopes exoskeleton robot for interactive gait rehabilitation. IEEE Trans Neural Syst Rehabil Eng 15(3):379-386

50. Verdaasdonk BW, Koopman HFJM, van der Helm FCT (2009) Energy efficient walking with central pattern generators: from passive dynamic walking to biologically inspired control. Biol Cybern 101(1):49-61

51. Walsh CJ, Endo K, Herr H (2007) A quasi-passive leg exoskeleton for load-carrying augmentation. International Journal of Humanoid Robotics 4(3):487-506

52. White O, Bleyenheuft Y, Ronsse R, Smith AM, Thonnard JL, Lefèvre P (2008) Altered gravity highlights central pattern generator mechanisms. J Neurophysiol 100(5):2819-2824

53. Winter DA (2009) Biomechanics and Motor Control of Human Movement, 4th edn. Wiley, New Jersey

54. Wolbrecht ET, Chan V, Reinkensmeyer DJ, Bobrow JE (2008) Optimizing compliant, model-based robotic assistance to promote neurorehabilitation. IEEE Trans Neural Syst Rehabil Eng 16(3): 286-297 